\title{
In situ Real-Time Monitoring of ITO Film under a Chemical Etching Process Using Fourier Transform Electrochemical Impedance Spectroscopy
}

Seok Hee Han ${ }^{1 \S}$, Jihun Rho ${ }^{1 \S}$, Sunmi Lee ${ }^{2}$, Moonjoo Kim¹, Sung Il Kim¹, Sangmee Park², Woohyuk Jang ${ }^{1}$, Chang Heon Lee ${ }^{1}$, Byoung-Yong Chang ${ }^{3 *}$, and Taek Dong Chung ${ }^{1,2,4^{*}}$

${ }^{1}$ Department of Chemistry, Seoul National University, Seoul 08826, South Korea

2 Program in Nano Science and Technology, Graduate School of Convergence Science and Technology, Seoul National University, Suwon-Si, Gyeonggi-do 16229, South Korea

${ }^{3}$ Department of Chemistry, Pukyong University, Busan 48513, South Korea

${ }^{4}$ Advanced Institute of Convergence Technology, Suwon-Si, Gyeonggi-do 16229, South Korea

$\S$ Both authors contributed equally to this work. 


\section{Content list}

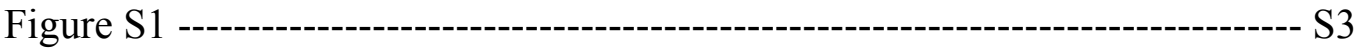

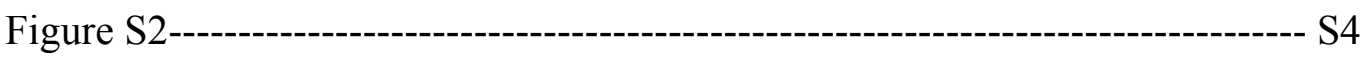



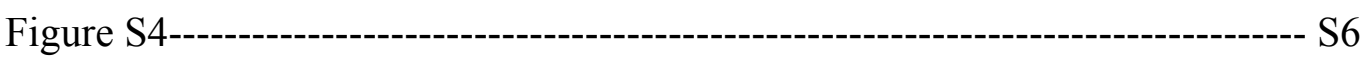

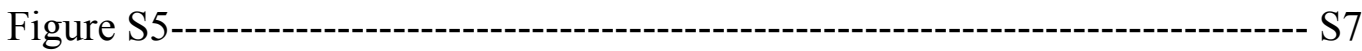

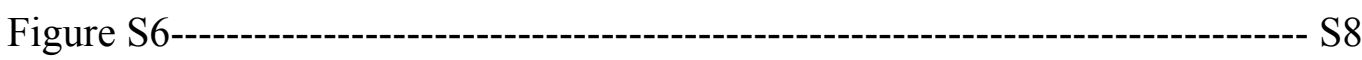

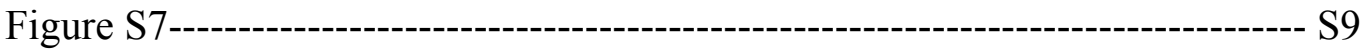

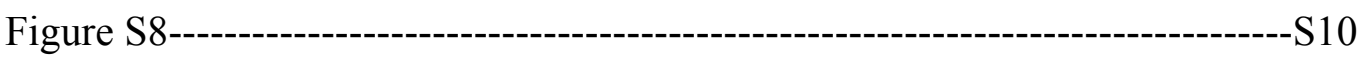

Figure S9--------------------------------------------------------------------------------------S11

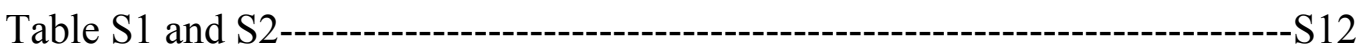

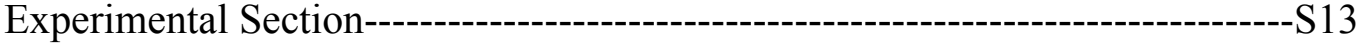

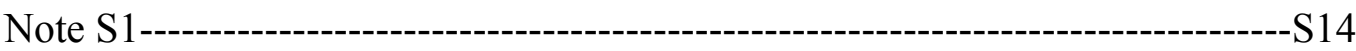

Movie S1---------------------------------------------------------------------------------------S15 
Figure S1. Two consecutive Nyquist plots measured immediately after immersion of the ITO microdisk array into the etchant solution via conventional FRA EIS analyzer. The etchant contained $3 \mathrm{M} \mathrm{FeCl}_{3}, 1 \mathrm{M} \mathrm{HCl}$, and its temperature was kept at $47^{\circ} \mathrm{C}$. (Frequency range $=50$ $\mathrm{kHz} \sim 1 \mathrm{~Hz}$, offset voltage $=0.490 \mathrm{~V}$ vs. $\mathrm{Ag} / \mathrm{AgCl}$ reference electrode, amplitude of sinusoidal wave $=5 \mathrm{mV}_{\mathrm{rms}}$ )

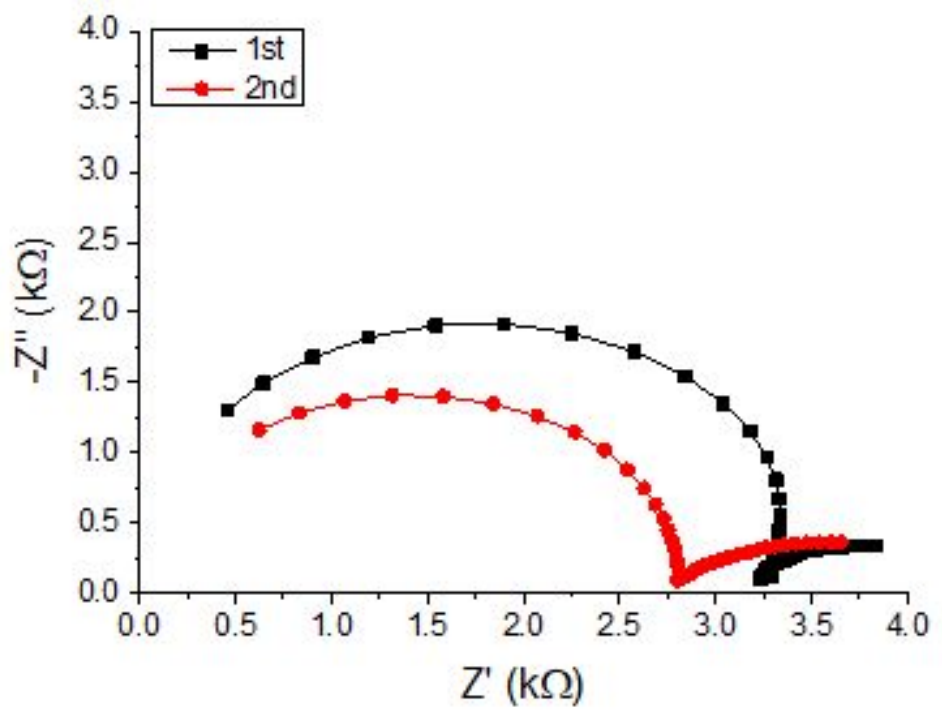


Figure S2. Model geometries of the ITO microdisk electrode for COMSOL simulation for one-electron transfer reaction, $\mathrm{Ox}+\mathrm{e} \leftrightarrow \mathrm{Red}$, with 2D axisymmetric configuration (A) when etching is still in process, and (B) when etching is finished. Red solid lines in (A) and (B) indicate the ITO electrode surface. The $5 \mu \mathrm{m}$-wall stands for the PR insulation layer. The scale of the length might be different from the real dimension. Concentration profiles of $\mathrm{Ox}$ species at $E_{\mathrm{eq}}(=0 \mathrm{~V})$ on cathodic scan when $C_{\mathrm{Ox}}{ }^{*}=C_{\mathrm{Red}}{ }^{*}=10 \mathrm{mM}(\mathrm{C})$ for geometry of (A), and (D) for geometry of (B). $\left(k^{0}=2.37 \times 10^{-6} \mathrm{~m} / \mathrm{s}, C_{\mathrm{dl}}=11.21 \mu \mathrm{F} / \mathrm{cm}^{2}, \alpha=0.5, D_{\mathrm{Ox}}=7.2 \times 10\right.$ ${ }^{-10} \mathrm{~m}^{2} / \mathrm{s}, D_{\mathrm{Red}}=6.7 \times 10^{-10} \mathrm{~m}^{2} / \mathrm{s}$, Scan rate $\left.=50 \mathrm{mV} / \mathrm{s}\right) k^{0}$ and $C_{\mathrm{dl}}$ values were calculated from the EIS measurement (see Note S1).

A

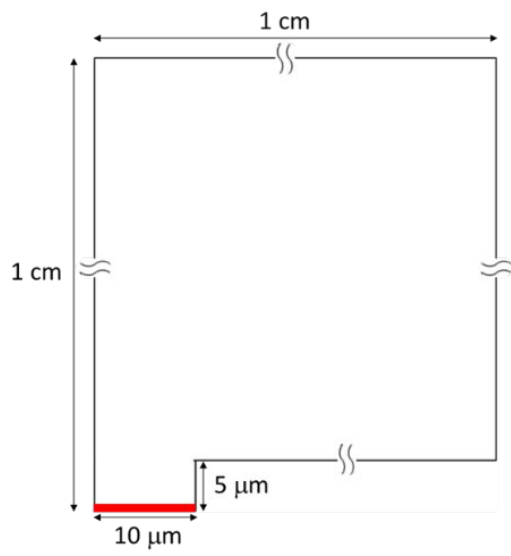

C

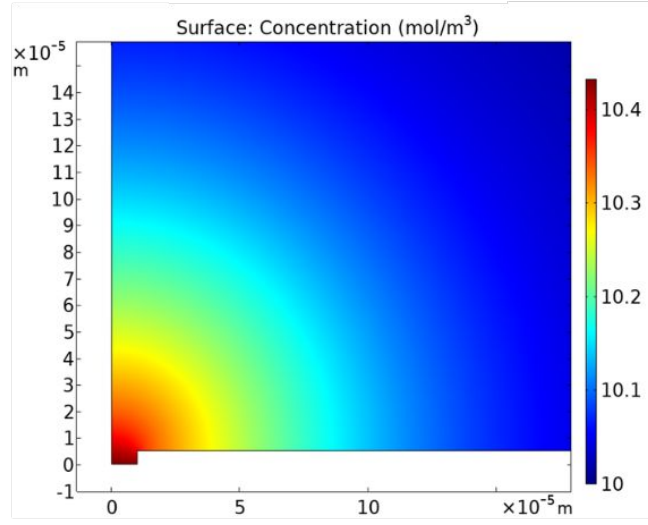

B

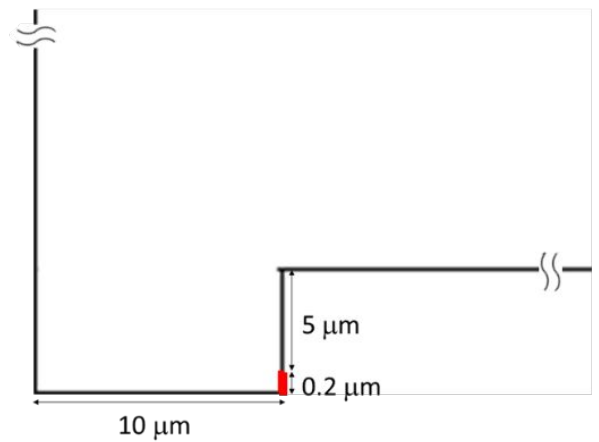

D

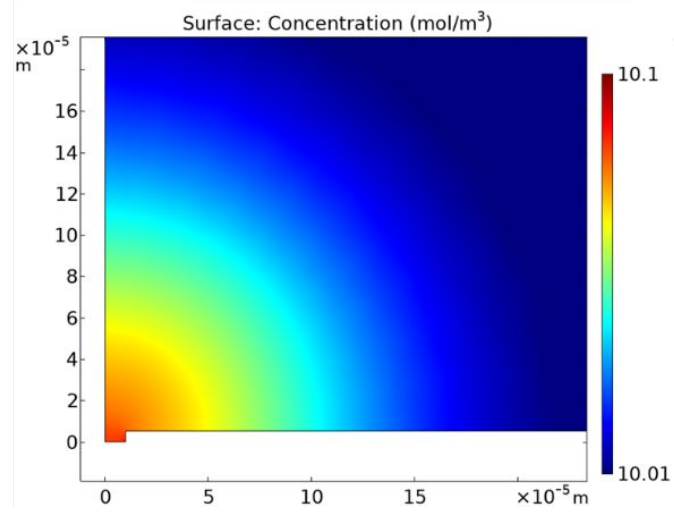


Figure S3. (A) Nyquist plot of the ITO microdisk under $3 \mathrm{M} \mathrm{FeCl}_{3}, 1 \mathrm{M} \mathrm{HCl}$ etchant at $47^{\circ} \mathrm{C}$ obtained from FT-EIS measurement when the etching is still in progress $(t=1 \mathrm{~min}$, frequency range $=50 \mathrm{kHz} \sim 1 \mathrm{~Hz}$ ). (B) Nyquist plots obtained from COMSOL simulation under the geometry of Figure S2A at $E_{\mathrm{eq}}$. Red symbol : $C_{\mathrm{Ox}}{ }^{*}=20 \mathrm{mM}, C_{\mathrm{Red}}{ }^{*}=0 \mathrm{mM}$, Black symbol: $C_{\mathrm{Ox}}{ }^{*}=C_{\mathrm{Red}}{ }^{*}=10 \mathrm{mM}$. (C) Cyclic voltammograms and (D) Nyquist plots obtained from COMSOL simulation with varying PR thickness under the geometry of Figure S2A. The vertical blue dashed line at $E=0 \mathrm{~V}$ indicates $E_{\text {eq }}$ in C. (scan rate $=50 \mathrm{mV} / \mathrm{s}$, frequency range $=100 \mathrm{kHz} \sim 1 \mathrm{~Hz})$ The same electrochemical parameters $\left(k^{0}, C_{\mathrm{dl}}, \alpha, D_{\mathrm{Ox}}, D_{\mathrm{Red}}\right)$ were used as in Figure S2.

A

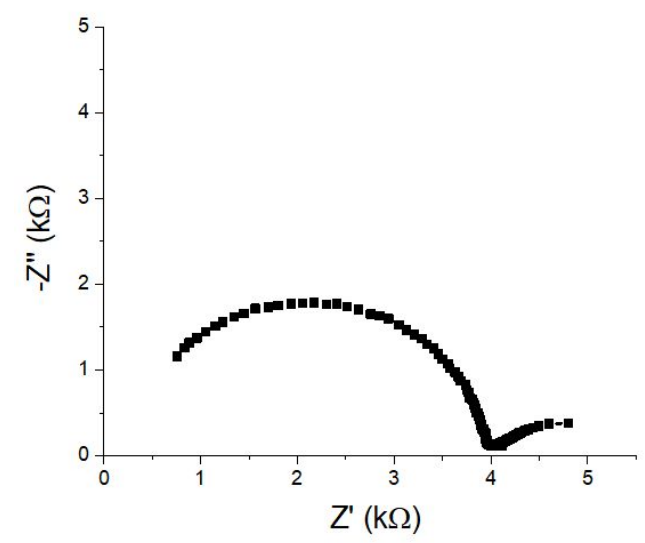

C

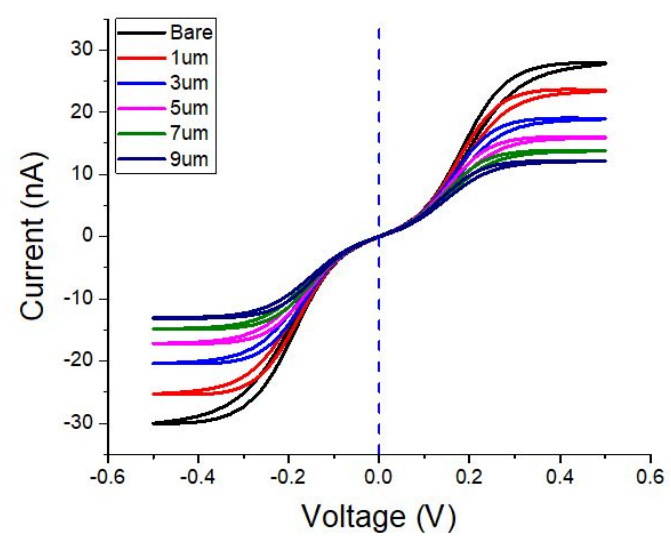

B

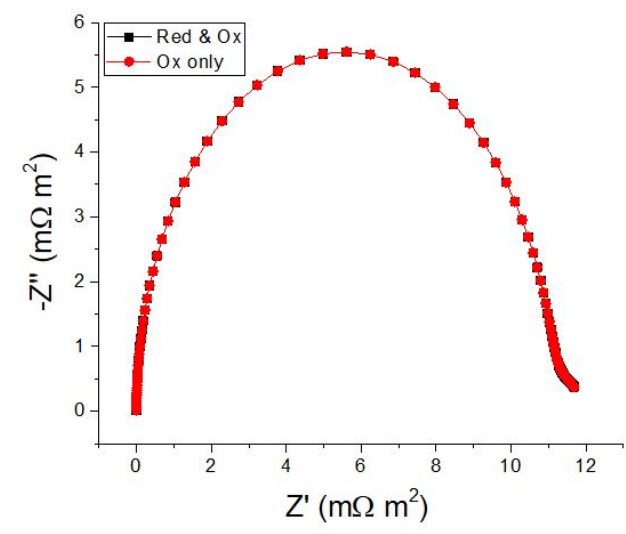

D

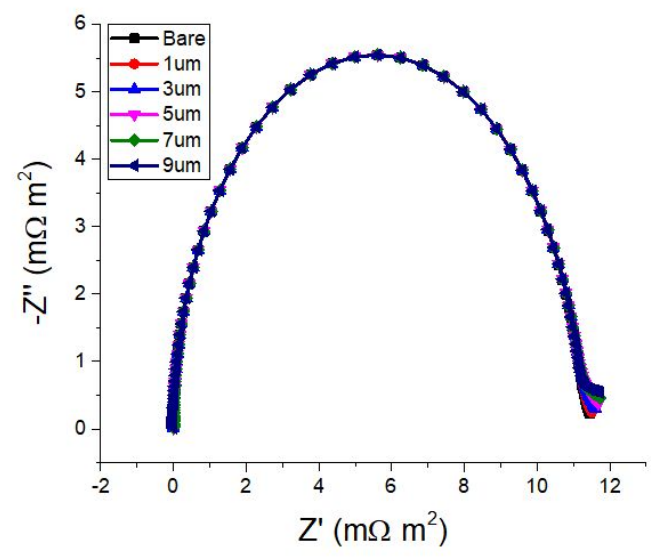


Figure S4. (A) The XRD spectrum of partially etched ITO thin film samples whose durations of etching are marked in blue color at the left. The corresponding crystalline facets are marked above each XRD peak. (B) Change of the XRD peak intensity ratio $I_{222} / I_{400}$ with the time duration of etching. The ITO samples were prepared by cutting the ITO-coated slide glass into individual rectangular pieces $(1 \mathrm{~cm} \times 1 \mathrm{~cm})$ with a diamond cutter.

A

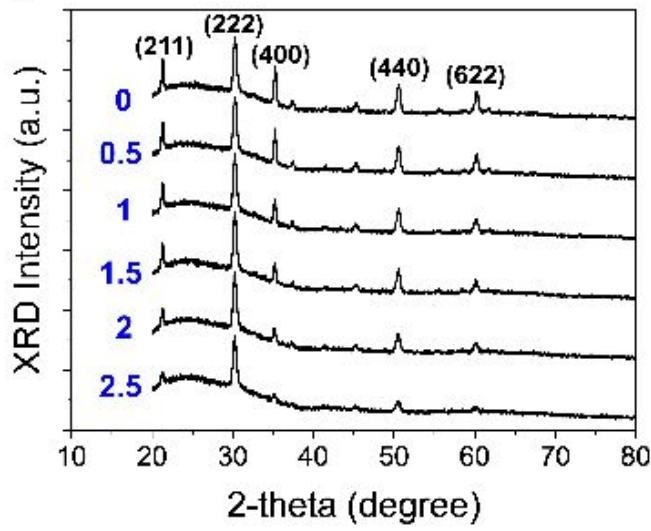

B

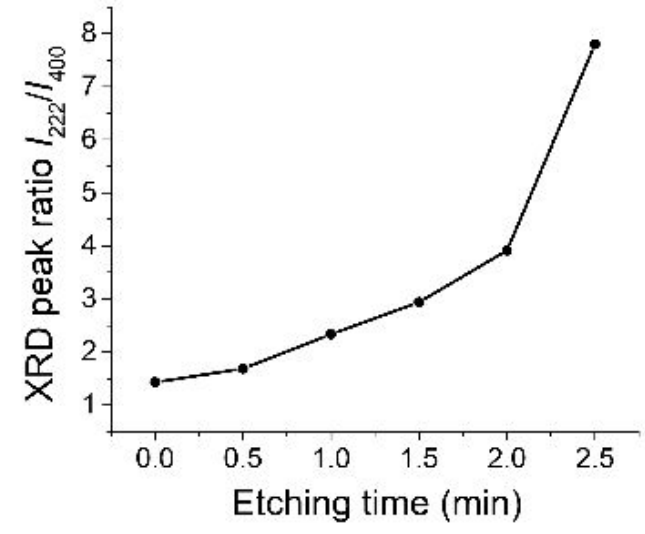


Figure S5. (A) FESEM image of a PR-covered ITO microdisk with $5.00 \mathrm{kX}$ magnification when the etching process was completed, and (B) the magnified image of the edge area marked with yellow dashed rectangle with $50.00 \mathrm{kX}$ magnification ratio.

A

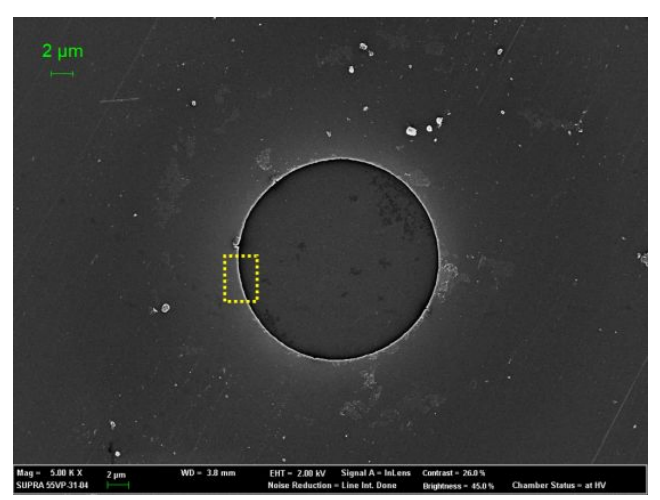

B

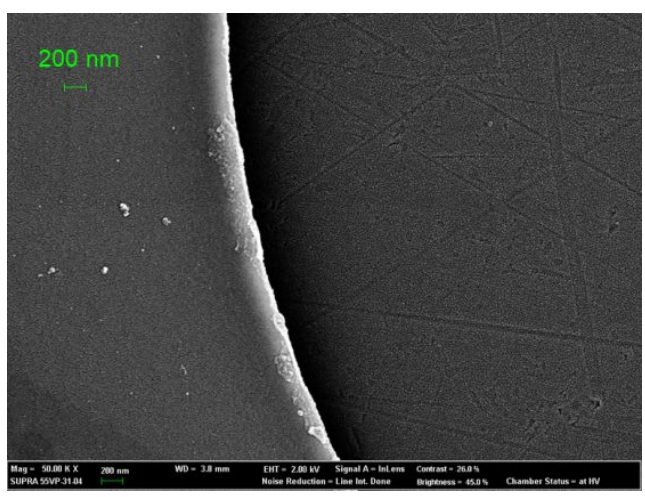


Figure S6. (A) Nyquist plot of the ITO microdisk under $3 \mathrm{M} \mathrm{FeCl}_{3}, 1 \mathrm{M} \mathrm{HCl}$ etchant at $47^{\circ} \mathrm{C}$ obtained from FT-EIS measurement after the etching is completed. ( $t=6 \mathrm{~min}$, frequency range $=50 \mathrm{kHz} \sim 1 \mathrm{~Hz}$ ). (B) Nyquist plots obtained from COMSOL simulation under the geometry of Figure S2B at $E_{\mathrm{eq}}$. Red symbol : ${C_{\mathrm{Ox}}}{ }^{*}=20 \mathrm{mM}, C_{\mathrm{Red}}{ }^{*}=0 \mathrm{mM}$, Black symbol : $C_{\mathrm{Ox}}{ }^{*}=C_{\mathrm{Red}}{ }^{*}=10 \mathrm{mM}$. (C) Cyclic voltammograms and (D) Nyquist plots obtained from COMSOL simulation with varying PR thickness under the geometry of Figure S2B. The vertical blue dashed line at $E=0 \mathrm{~V}$ indicates $E_{\text {eq }}$ in C. (scan rate $=50 \mathrm{mV} / \mathrm{s}$, frequency range $=100 \mathrm{kHz} \sim 1 \mathrm{~Hz})$ The same electrochemical parameters $\left(k^{0}, C_{\mathrm{dl}}, \alpha, D_{\mathrm{Ox}}, D_{\mathrm{Red}}\right)$ were used as in Figure $\mathrm{S} 2$.

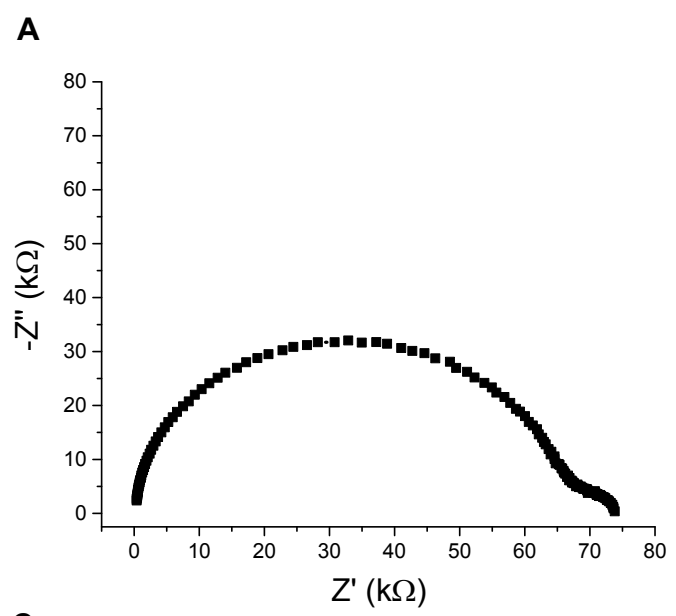

C

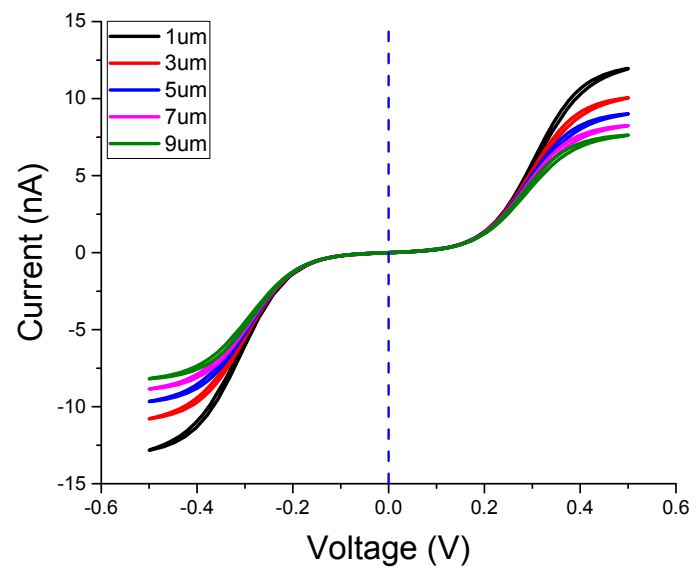

B



D

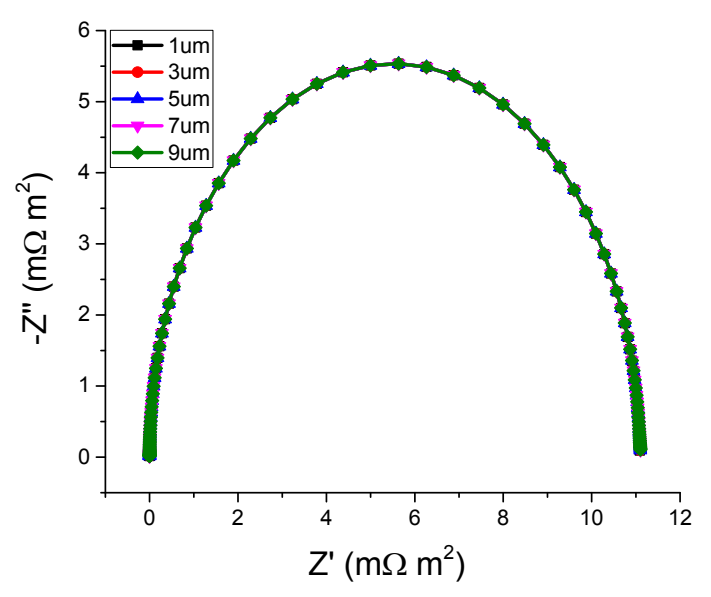


Figure S7. Nyquist plots of ITO microdisk electrode of the model geometries in Figure S3B and S6B, computed from COMSOL simulation before (black symbols) and after (red symbols) the etching process is completed, where (A) $C_{\mathrm{Ox}}{ }^{*}=C_{\mathrm{Red}}{ }^{*}=10 \mathrm{mM}$, and (B) $C_{\mathrm{Ox}}{ }^{*}=20$ $\mathrm{mM}, C_{\mathrm{Red}}{ }^{*}=0 \mathrm{mM}$.

A

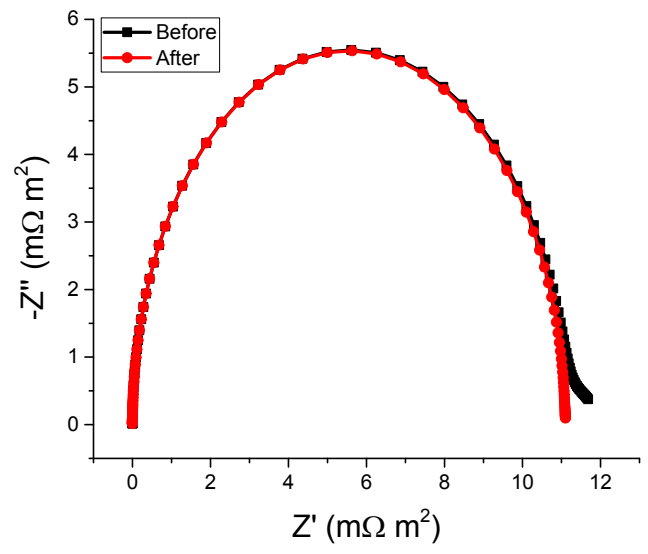

B

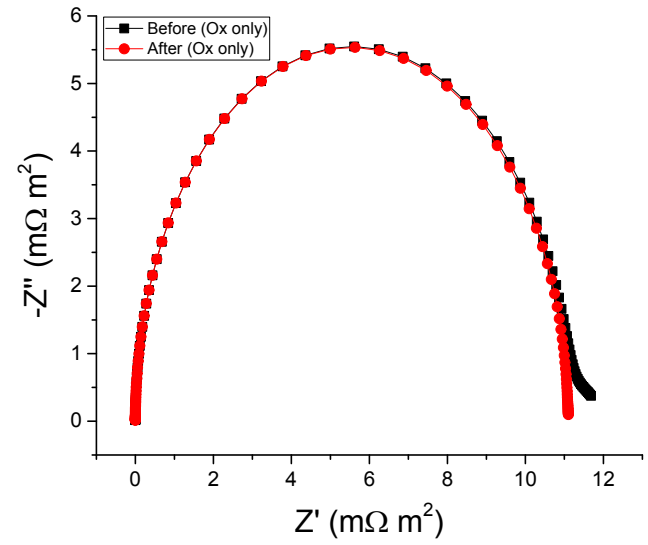


Figure S8. (A) FESEM image of an etched ITO microdisk whose PR layer was removed with acetone after the etching process. FESEM images of the edge area of the same sample with magnification ratio of (B) $50.00 \mathrm{kX}$ and (C) $100.00 \mathrm{kX}$. (D) Tilted FESEM image for focusing on the remaining sidewall area.
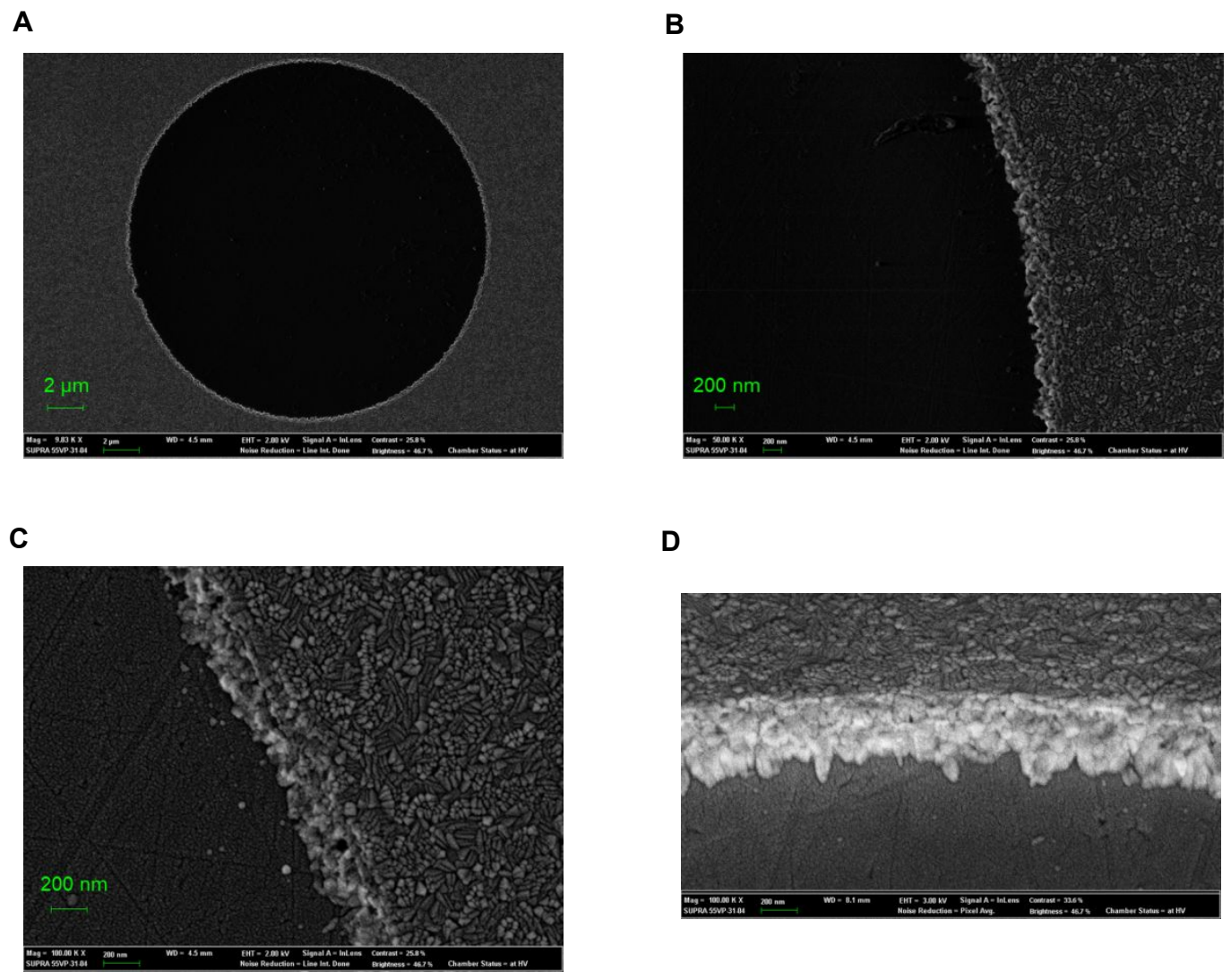

\section{D}

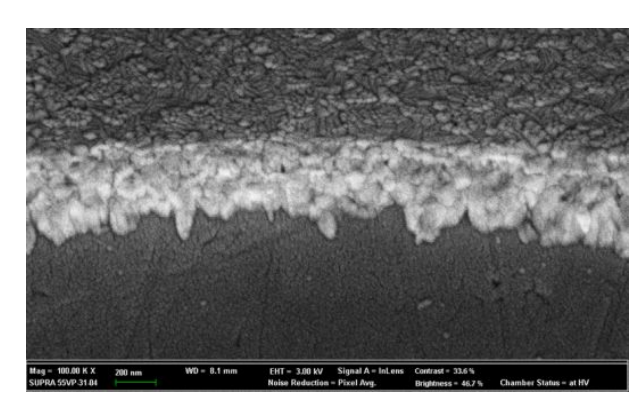


Figure S9. Optical microscopic images of (A) the five ITO interdigitated array (IDA) samples chemically etched with simultaneous FT-EIS monitoring, and (B) the other five chemically etched for 10 min without FT-EIS monitoring. The etching was carried out with 3 $\mathrm{M} \mathrm{FeCl}_{3}$ with $1 \mathrm{M} \mathrm{HCl}$ solution at $47^{\circ} \mathrm{C}$. All the images were taken after removal of photoresist (PR) after the etching process. The red dashed circles indicate the areas with damaged or broken patterns.

A

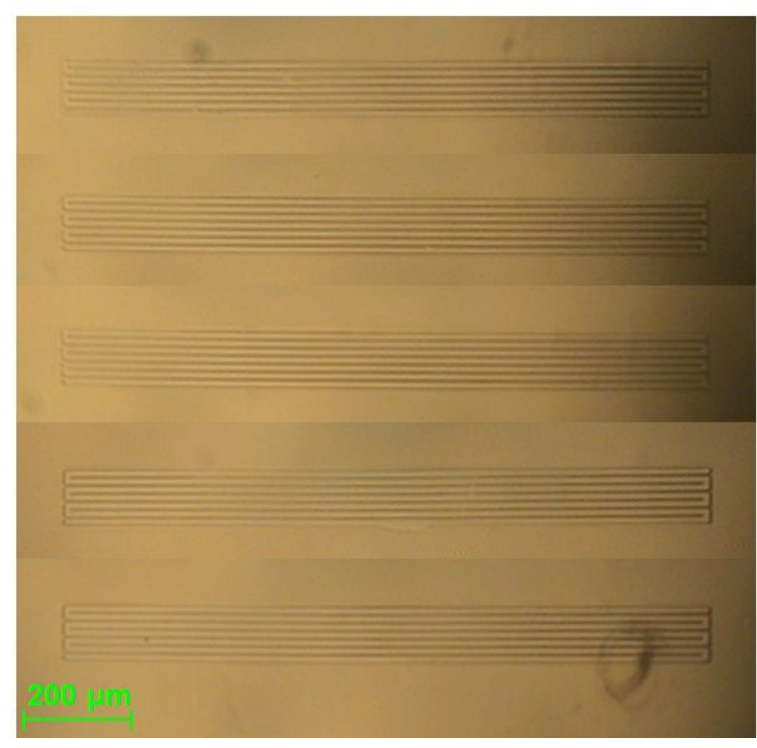

B




Table S1. Near-surface atomic composition of Sn and In obtained from EDS analysis for ITO films with various etch durations

\begin{tabular}{lllllll}
\hline & \multicolumn{6}{c}{ Etch duration (min) } \\
\cline { 2 - 7 } & 0 & 0.5 & 1 & 1.5 & 2 & 2.5 \\
\hline \multicolumn{7}{l}{ Relative composition (\%) } \\
$\mathrm{In}$ & 23.27 & 21.01 & 18.56 & 16.77 & 10.70 & 7.70 \\
$\mathrm{Sn}$ & 2.23 & 2.02 & 1.80 & 1.67 & 1.09 & 0.81 \\
$\mathrm{Sn} / \mathrm{In}$ & 0.0958 & 0.0961 & 0.0970 & 0.0996 & 0.1019 & 0.1052 \\
\hline
\end{tabular}

Table S2. Time required for completion of etching for the ITO IDA samples in Figure 4B.

\begin{tabular}{|c|c|c|c|c|c|}
\hline Sample No. & $\# 1$ & $\# 2$ & $\# 3$ & $\# 4$ & $\# 5$ \\
\hline Etching duration (min) & 5.50 & 5.07 & 4.90 & 4.60 & 5.13 \\
\hline
\end{tabular}




\section{Experimental Section}

Materials and Reagents. All chemicals were used without further purification. ITO coated glass (TIXZ003, $200 \mathrm{~nm}$ thick ITO on $0.7 \mathrm{~mm}$ thick borosilicate glass, $\sim 10 \mathrm{ohms} / \mathrm{sq}$ of surface resistivity) fabricated by magneton sputtering at $300{ }^{\circ} \mathrm{C}$ was purchased from Techinstro. Hydrochloric acid $(\mathrm{HCl}, 37 \%)$, ferric chloride hexahydrate $\left(\mathrm{FeCl}_{3} \cdot 6 \mathrm{H}_{2} \mathrm{O}, 97 \%\right)$, potassium hexacyanoferrate(III) $\left(\mathrm{K}_{3}\left[\mathrm{Fe}(\mathrm{CN})_{6}\right]\right)$, potassium hexacyanoferrate(II) trihydrate $\left(\mathrm{K}_{4}\left[\mathrm{Fe}(\mathrm{CN})_{6}\right] \cdot 3 \mathrm{H}_{2} \mathrm{O}\right)$ were purchased from Aldrich.

Fabrication of ITO Micropattern Samples. The ITO micropatterns (i.e. microdisk array, interdigitated array pattern) coated with AZ4620 positive photoresist (PR; AZ4620, Clariant, Switzerland) were fabricated by following a general photolithography procedure. TIXZ003 ITO coated glasses $(75 \mathrm{~mm} \times 25 \mathrm{~mm}, 0.7 \mathrm{~mm}$ thick, Techinstro, USA) were used as substrates. The substrate was rinsed with acetone, ethanol, and deionized (DI) water (NANOpure Diamond, Barnstead, USA) several times. After removing the moisture on the surface with an air blower, the cleaned ITO coated glass was dehydrated on a hot plate at $150{ }^{\circ} \mathrm{C}$ for $10 \mathrm{~min}$ and then cooled to room temperature. The slide was then spin-coated (YS-100MD, Won Corp., Korea) with hexamethyldisilazane (HMDS; Clariant, Switzerland) at $6000 \mathrm{rpm}$ for $30 \mathrm{~s}$. After soft baking the $\mathrm{PR}$ on a hot plate at $120^{\circ} \mathrm{C}$ for $90 \mathrm{~s}$, it was then coated with $\mathrm{AZ} 4620$ at 6000 rpm for $30 \mathrm{~s}$. After soft baking the PR on a hot plate at $100{ }^{\circ} \mathrm{C}$ for $90 \mathrm{~s}$, the substrate was cooled to room temperature and aligned under a pattern mask. The PR on the slide was exposed to UV light $(365 \mathrm{~nm})$ with an intensity of $21 \mathrm{~mW} \mathrm{~cm}^{-2}$ for $13 \mathrm{~s}$ (MDE-4000, Midas, Korea) and AZ $400 \mathrm{~K}$ developer (Clariant, Switzerland) for $150 \mathrm{~s}$. The PR-coated substrate was then washed with DI water, and the PR was hard-baked on a hot plate at $120^{\circ} \mathrm{C}$ for $15 \mathrm{~min}$.

Investigation of Near-surface Characteristics of Etched ITO Samples. Field emission scanning electron microscopy (FESEM) (SUPRA 55VP, Carl Zeiss, Germany) was used for observing surface morphologies of ITO substrates at several points during the chemical etching process. High resolution X-ray diffraction (HRXRD) (SmartLab, Rigaku, Japan) and energydispersive X-ray spectroscopy (EDS) (SUPRA 55VP, Carl Zeiss, Germany) were used to examine the crystallographic change and atomic composition of the etched ITO surface. 
Note S1. Calculation of $k^{0}$ and $C_{\mathrm{dl}}$ from EIS measurement.

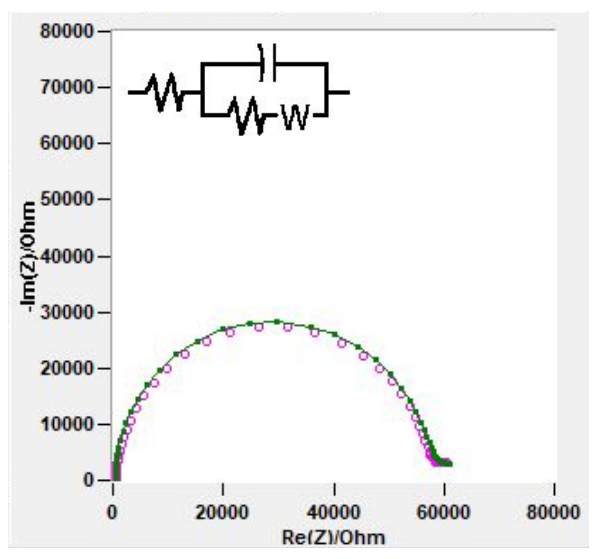

\begin{tabular}{|c|l|l|}
\hline Parameters & \multicolumn{1}{|c|}{ Value } & \multicolumn{1}{|c|}{ Relative S.D. } \\
\hline$R_{s}$ & $508.19 \Omega$ & 0.0267 \\
\hline$R_{c t}$ & $56.171 \mathrm{k} \Omega$ & 0.0203 \\
\hline$C_{d l}$ & $22.015 \mathrm{nF}$ & 0.0160 \\
\hline
\end{tabular}

In order to calculate standard rate constant $k^{0}$ and electrical double layer capacitance $C_{\mathrm{dl}}$ to be used in COMSOL simulation, the impedance measurement was conducted as described above using $10 \mathrm{mM}\left[\mathrm{Fe}(\mathrm{CN})_{6}\right]^{3-/ 4-}$ with $100 \mathrm{mM}$ phosphate buffer $(\mathrm{pH}=7.4)$ near $E^{0}$ on $0.5 \mathrm{~mm}$ diameter ITO macroelectrode covered with PR insulation layer. The $R_{\mathrm{ct}}$ and $C_{\mathrm{dl}}$ were extracted from the EIS data as summarized in the table above. According to eqn (1) below, $k^{0}$ can be calculated from $R_{\mathrm{ct}}$ and bulk concentration of the redox species $C^{*}$, where $\mathrm{R}=8.3145 \mathrm{~J}$ $\mathrm{K}^{-1} \mathrm{~mol}^{-1}, \mathrm{~T}=293.15 \mathrm{~K}$.

$$
\begin{gathered}
i_{0}=\frac{R T}{F R_{c t}}=F A k^{0} C_{O x}{ }^{*(1-\alpha)} C_{R e d}{ }^{* \alpha}=\mathrm{FAk}^{0} C^{*}\left(\text { where } C_{O x}{ }^{*}=C_{R e d}{ }^{*}=C^{*}\right) \\
k^{0}=\frac{R T}{F^{2} R_{c t} A C^{*}}
\end{gathered}
$$

This equation can be reexpressed as eqn (2), which gives $k^{0}=2.37 \times 10^{-6} \mathrm{~m} / \mathrm{s}$. In consideration of the electrode area, the double layer capacitance can be calculated as $C_{\mathrm{dl}}=$ $11.21 \mu \mathrm{F} / \mathrm{cm}^{2}$. 
Movie S1. Continuous monitoring of equivalent circuit parameters fitted from real-time FTEIS data during the ITO etching process. 